\title{
Criticality in quark-gluon systems far beyond thermal and chemical equilibrium
}

\author{
Fu Jinghua ${ }^{1}$, Meng Ta-chung ${ }^{1,2}$, R. Rittel ${ }^{2}$, and K. Tabelow ${ }^{2}$ \\ ${ }^{1}$ Institute of Particle Physics, CCNU, 430079 Wuhan, China \\ ${ }^{2}$ Institut für Theoretische Physik, FU-Berlin, 14195 Berlin, Germany \\ e-mail: fujh@iopp.ccnu.edu.cn; meng@iopp.ccnu.edu.cn; meng@physik.fu-berlin.de; \\ rittel@physik.fu-berlin.de; tabelow@physik.fu-berlin.de
}

\begin{abstract}
Experimental evidence and theoretical arguments for the existence of self-organized criticality in systems of gluons and quarks are presented. It is observed that the existing data for high-transverse-momentum jet-production exhibit striking regularities; and it is shown that, together with first-principle considerations, such regularities can be used, not only to probe the possible compositness of quarks, but also to obtain direct evidence for, or against, the existence of critical temperature and/or critical chemical potential in quark-gluon systems when hadrons are squeezed together.
\end{abstract}

The question whether a "new state of matter", QGP (quark-gluon plasma), can be created in laboratories has been raised soon after physicists found that the fundamental building blocks of hadronic matter are quarks and gluons, and that their (color) interactions can be described by Quantumchromodynamics (QCD). Transitions between such "new states" and normal hadronic states are usually knownt, 2 as "phase transitions" in analogy to those discussed in connection with critical phenomena in Equilibrium Thermodynamics; and these transitions are expected to occur at a given (critical) temperature and/or at a given (critical) chemical potential. While a number of devoted high-energy nucleus-nucleus collision experiments have been performed, no direct signals for the existence of QGP has yet been found 1 . 
With the help of high-energy proton-antiproton (Tevatron) and heavy-ion (RHIC) colliders, it is now possible to measure high-transverse-momentum $\left(p_{T}\right)$, or -energy $\left(E_{T}\right)$-jets due to "hard" subhadronic scattering processes. It is anticipated2国 that such experiments would yield useful information, not only on the structure of hadrons, but also on the possible compositness of quarks $\mathrm{B}_{\text {l }}$. However, due to the flexibility of the parton distribution functions (PDF's) it is extremely difficult for experimentalists to draw definite conclusions from their data - independent of the accuracy of their experiments.

In this paper: We report on the observation of striking regularities, in particular the observation of fingerprints of self-organized criticality (SOC) induced high- $E_{T}$ jet production processes. We show that these observations can be used, not only in probing the compositness of quarks, but also as direct tests for the existence of thermal and/or chemical equilibrium, and thus as direct tests for the existence of critical temperature and/or critical chemical potential for quark-gluon system, 晅 when hadrons are squeezed together.

We recalle: The characteristic properties of the gluons - in particular the direct gluon-gluon coupling prescribed by the QCD-Lagrangian, the confinement, and the nonconservation of gluon-numbers, show that systems of interacting soft gluons should be considered as open dynamical complex systems which are in general far from thermal and/or chemical equilibrium. Taken together with the observations畸 made by Bak, Tang and Wiesenfeld (BTW), this strongly suggests the existence of self-organized criticality (SOC, i.e. criticality without the need of fine tuning of any external parameter such as temperature) and thus the existence of BTW-avalanches in such systems.

We also recall: A small part of such BTW-avalanches manifest themselves in form of color-singlet gluon clusters $\left(c_{0}^{\star}\right)$ which can be readily examined 9 㽖 experimentally in inelastic diffractive scattering processes. This is because the interactions between the struck $c_{0}^{\star}$ and any other color-singlets are of Van der Waal's type which are similar to those between nucleons in nucleus (that is, they are much weaker than color forces at distances of hadron-radius). In order to check the existence and the properties of the $c_{0}^{\star}$ 's, a systematic data-analysis has 
been performed , the result of which shows that the distribution $D_{S}(S)$ of the size $S$, and the distribution $D_{T}(T)$ of the lifetime $T$ of such $c_{0}^{\star}$ 's indeed exhibit power-law behaviors [namely $D_{S}(S) \propto S^{-\mu}, D_{T}(T) \propto T^{-\nu}$, where $\mu$ and $\nu$ are positive real constants]. Such characteristic features are known as "the fingerprints of $S O C$ ", 1 . In particular, taking into account, that in inelastic diffractive scattering, the size $S$ of the struck $c_{0}^{\star}$ is proportional to the directly measurable quantity $x_{P}$, which is the energy fraction carried by "the exchanged colorless object" in such scattering processes, it is found from the existing datal that $D_{S}\left(x_{P}\right) \propto x_{P}^{-\mu}$, where $\mu=1.95 \pm 0.12$, that is $\mu \approx 2$.

By considering inelastic diffractive scattering 6 , we were able to check — and only able to check (the existence and the properties of) the color-singlet gluon-clusters. Due to the experimentally observed SU(3) color-symmetry, most of such gluon-clusters are expected to carry color quantum numbers; that is, they are color-multiplets (instead of singlets) which will hereafter be denoted by $c^{\star}$ s. But in accordance with the experimentally confirmed characteristic features of the BTW theory, the existence of SOC fingerprints in gluon systems should not depend on the dynamical details of their interactions - in particular the intrinsic quantum numbers they exchange during the formation process. This means in particular, that the size $(S)$ distribution $D_{S}(S)$ and the lifetime $(T)$ distribution $D_{T}(T)$ of the $c^{\star}$ 's are expected to have, not only the same power-law behavior, but also the same power as those of their color-singlet counterparts observed in diffractive scattering processes 6 :

Having in mind that quarks can be knocked-out of protons by projectiles in deep-inelastic scattering processes 8 where large momentum transfer between projectile and target takes place, it is not difficult to imagine that colored gluon-clusters $\left(c^{\star}\right.$ 's) can also be "knocked out" of the mother proton $(p)$ by a projectile provided that the corresponding transfer of momenta is large enough (Here the knocked-out $c^{\star}$ 's may or may not have "color lines" connected to the remnant of the proton - depending on the final state of the struck $c^{\star}$ after it has absorbed the object which delivers the momentum transfer).

In this paper, we show that there is indeed experimental evidence for such knock-out processes. First, we point-out a striking regularity: The differential cross section $d^{2} \sigma / d E_{T} d \eta$ 
(for $\eta \approx 0$ ) data 12 for inclusive high- $E_{T^{-}}$jet production processes $p+\bar{p} \rightarrow$ jet $+X$ behaves like $E_{T}^{-\alpha}$, where $\alpha=5,7$ and 9 in the lower, medium, and higher $E_{T}$-region, respectively 13 , as can be readily seen in the figure. Next we show that this behavior is due to the probability distribution of the knocked-out colored gluon clusters and the phase-space factors related to the processes in which the large transverse momentum $p_{T}\left(\approx E_{T}\right)$ is transferred.

We begin our discussion with the experimental observation 14 , 2 that the inclusive high-

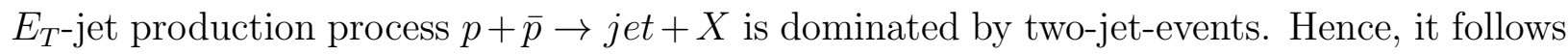
from the SOC-picture in which every jet corresponds to a knocked-out colored gluon clusters $c^{\star}$, that the invariant cross section $E d^{3} \sigma / d p^{3}$ is expected to be approximately proportional to the square of the probability $D_{S}$ to find a $c^{\star}$. The fact that the jet mass $m$ is negligible small $\left(m^{2}=E_{T}^{2}-p_{T}^{2} \approx 0\right)$ implies that $E_{T}$ is approximately the energy carried by the knocked-out $c^{\star}$. Having in mind that田 the size $S$ of BTW-avalanches is directly proportional to the dissipative energy it carries, we are immediately led to

$D_{S}\left(E_{T}\right) \propto E_{T}^{-\mu}, \quad \mu \approx 2$.

Hence, we expect to see a factor $E_{T}^{-4}$ in the invariant cross section $E d^{3} \sigma / d p^{3}$ which is related to the measured quantity shown in the Figure as follows: $2 \pi E_{T}^{-1} d^{2} \sigma / d E_{T} d \eta=E d^{3} \sigma / d p^{3}$.

We now show that the phase-space factors in $E d^{3} \sigma / d p^{3}$ which corresponds to the different possibilities for a $c^{\star}$ to receive a large transverse momentum $p_{T}\left(\approx E_{T}\right)$ are also powers in $E_{T}$. In order to see this, we recall the well-known observation made by Rutherford 15 in which the size and the location of the effective scatterer in an atom is probed by examing largemomentum-transfer scattering, and to recall a less-known observation made by Williams 16 in which the following has been pointed out: Ordinary space-time concepts are indeed very useful for the semiclassical description of high-energy scattering processes, provided that the de Broglie wave-length of the projectile is short compared to the linear dimension of the scattering field, and provided that the corresponding momentum-transfer which determines the deflection is not smaller than the disturbance allowed by the Uncertainty Principle. The above-mentioned phase-space factors can be estimated by making use of the Uncertainty 
Principle and these two observation, 1516 . To be more precise, when we choose the $z$-axis as the collision axis and consider the momentum-transfer in (or near) the $x y$-plane, the chance for two objects (c.m. of $p$ and $\bar{p}$ and/or their constituents) moving parallel to the $z$-axis to come so close to each other in the $x y$-plane such that an amount of transverse momentum $p_{T}$ can be transfered is approxiamtely proportional to the size of the corresponding phase-space $\Delta x \Delta y \propto\left(\Delta p_{x}\right)^{-1}\left(\Delta p_{y}\right)^{-1} \propto p_{T}^{-1} p_{T}^{-1} \propto E_{T}^{-2}$. The same procedure can be applied to the time-degree-of-freedom where we obtain $\Delta t \propto(\Delta E)^{-1} \propto\left(\Delta E_{T}\right)^{-1} \propto E_{T}^{-1}$, because not only a large momentum $p_{T}$ but also a corresponding large energy $\left(E=E_{T} \approx p_{T}\right)$ is transfered.

We recalla following preexisting constituents: (i) the valence quarks $q_{v}\left(\bar{q}_{v}\right)$, which dominate the region $0.2<x<1$, where $x$ denotes the fraction of protons momentum they carry, (ii) the sea quark-antiseaquark pairs $q_{s} \bar{q}_{s}$, which dominate the $10^{-2}<x<0.2$ region, and (iii) the gluon-clusters $c^{\star}$ 's, which dominate the $x<10^{-2}$ region. Hence, energy- and momentum-conservation, and the above-mentioned properties of these constituents dictate that we should see three different kinds of processes which dominate three distinct kinematical regions characterized by the values of $E_{T}$ :

(a) In the low- $E_{T}$-region the dominating contribution comes from One-Step (OS) processes in which a $c^{\star}$ of size $S \propto E_{T}$ in $p$ collides with a $c^{\star}$ of size $S \propto E_{T}$ in $\bar{p}$, where a transverse momentum of the order $p_{T}$ is exchanged in the $c^{\star}-c^{\star}$ scattering.

(b) In the medium- $E_{T}$-region the dominating contribution comes from Two-Step (TS) processes in which at least one of the quarks or antiquarks from the sea is involved in the first step. We call hereafter such processes TSS-processes. [Because of the obvious symmetry in $p$ and $\bar{p}$, we discuss here only $c^{\star}$ in $\bar{p}$ and $q_{s}\left(\right.$ or $\left.\bar{q}_{s}\right)$ in $p$, but not the other possible combination which also contributes.] TSS-processes can take place in two different ways (cf. Table): (b1) A $c^{\star}$ of size $S \propto E_{T}$ in $\bar{p}$ receives a transverse momentum transfer of order $p_{T}$ from a $q_{s}$ of $p$ in the first step and manifests itself as a high- $E_{T}$-jet. In the second step the final state of the scattered $q_{s}$ which is in general space-like, encounters one of the $c^{\star}$ s in its surroundings transfering its transverse momentum of order $p_{T}$, and thus give 
birth to the second (approximately zero-mass) high- $E_{T^{-j}}$ jet. (b2) A $q_{s}$ in $p$ meets a $q_{s}$ in $\bar{p}$, exchange a transverse momentum of order $p_{T}$, and each of the scattered objects encounters a corresponding $c^{\star}$ and thus give birth to two (approximately zero-mass) high- $E_{T}$-jets.

(c) In the high- $E_{T}$-region the dominating contribution comes from Two-Step (TS) processes in which at least one of the available valence quarks or antiquarks are involved in the first step. We call hereafter such processes TSV processes, which can also take place in two different ways: (c1) A $c^{\star}$ of size $S \propto E_{T}$ in $\bar{p}$ receives a transverse momentum-transfer of the order $p_{T}$ from a $q_{v}$ in $p$ in the first step, and thus manifest itself as a high- $E_{T}$-jet. In the second step, the scattered final state $q_{v}$ (which is in general space-like) encounters a $c^{\star}$ of size $S \propto E_{T}$ transfering its transverse momentum of order $p_{T}$ and become the second high- $E_{T}$-jet. $(\mathbf{c 2}) \mathrm{A} q_{v}$ in $p$ meets a $\bar{q}_{v}$ (or $q_{s}$ or $\left.\bar{q}_{s}\right)$ in $\bar{p}$, exchange $p_{T}\left(\approx E_{T}\right)$ in the first step, and each of the scattered objects encounters a suitable $c^{\star}$ in its surrounding and thus give birth to two high- $E_{T}$ jets.

Note that in the quantitative estimation of the phase-space-factors the following empirical facts䁬国 as well as their logical consequences have to be taken into account.

First, the $q_{s}$ 's, the $\bar{q}_{s}$ 's, and the gluons organized in form of $c^{\star}$ 's can be found everywhere inside the $p / \bar{p}$. Hence the chance for a $c^{\star}$ (or $q_{s}$ or $\bar{q}_{s}$ ) of $p$ to meet a $c^{\star}$ (or $q_{s}$ or $\bar{q}_{s}$ ) of $\bar{p}$ in a cell of the size $\Delta x \Delta y$ should be the same as that for the c.m. of $p$ and that of $\bar{p}$ to be in a phase-space-cell of the same size. The size of this two-dimensional phase space is $\Delta x \Delta y \propto p_{T}^{-2} \propto E_{T}^{-2}$.

Second, Two-Step processes (both TSS and TSV) can take place only when there are suitable $c^{\star}$ 's in the surroundings immediately after the first step. The probability for having sufficient $c^{\star}$ s around, whenever and wherever two constituents meet during the $\bar{p} p$-collision is guaranteed when the c.m. of $p$ meets that of $\bar{p}$. Hence, we can use the latter condition to perform the estimation. This means, the phase-space factor in the time-dimension for TSS or TSV to take place is $(\Delta t)^{2} \propto E_{T}^{-2}$.

Third, since there are only three $q_{v}$ 's in $p$, and they can be anywhere inside $p$, the chance for a $q_{v}$ to take part in the first step of a TS process (i.e. for TSV) to occur is expected to 
be smaller than that in TSS processes. Here, we need to find for example the probability for a $q_{v}$ in $p$ to meet either any $q_{s}$ or any $\bar{q}_{s}$ or one of the three $\bar{q}_{v}$ 's in $\bar{p}$. By using the same method, we readily see that it implies that an extra phase-space-factor $\Delta x \Delta y \propto E_{T}^{-2}$ should be taken into account.

The result of this estimation, summarized in the Table, explicitly shows that the phasespace-factors for such high- $E_{T}$-jet production processes can always be written as $E_{T}^{-\varphi}$ where $\varphi=2,4$ and 6 for OS and TSS and TSV processes respectively. Furthermore, since the phase-space-factors and the probability for obtaining $c^{\star}$ s of a given size are independent of one another, we are immediately led to the conclusion

$\frac{d^{2} \sigma}{d E_{T} d \eta} \propto E_{T}^{-\alpha}$,

where $\alpha=\varphi-1+2 \mu$, which is 5,7 , and 9 , respectively (for $\mu \approx 2$ ).

Comparison with the obtained result given in Eq.(2) and the existing data 12 is shown in the Figure.

In conclusion, the results presented in Eqs.(11) and (2) and in the Figure show that highenergy transverse-energy $\left(E_{T}\right)$ jet-production experiments can be used to find the solutions of the following longstanding puzzels:

(I) The observation䀦 that self-organized criticality (SOC) proposed by Bak, Tang and Wiesenfeld (BTW) can be found in complex systems as diverse as earthquakes, sandpiles, biological evolution and even stock markets, have triggered the question: "How general and/or how basic is SOC?"; in particular, "Is this kind of behavior observed in open complex systems so universal and so basic that SOC should exist also at the fundamental level of matter - in the world of quarks and gluons where the interactions are color forces prescribed by QCD?" While experimental indications for the existence of color-singlet gluon-clusters in form of BTW-avalanches have already been found 8 , the existence of their colored counterparts - without which the universality of SOC at the fundamental level of matter and the independence of dynamical details which is intimately related to the robustness of its occurrence could not be demonstrated - remains to be shown. With the results summarized in 
Eqs. (11) and (2), and in the Figure, we can now answer the above-mentioned questions in the affirmative.

(II) Large-momentum-transfer scattering has been very helpful in studying the structure of atoms 15 and that of nucleons 8 ; this idea of Rutherford 15 has also been applied to probe the possible compositness of quarks. In fact, the existing data 12 for high- $E_{T^{-}}$jet-production have been compared 311, 12 with the results obtained from pQCD and the existing PDF's2月. Due to the flexibility of the PDF's, however, the question "Is there experimental indication for compositness because the data exhibit a behavior which differs from the predictions made on the basis of the present theoretical understanding?" could not be answered in a unique way. In this paper, we report on the observation of a striking regularity, and we show that the differential cross section $d^{2} \sigma / d E_{T} d \eta$ for $p+\bar{p} \rightarrow$ jet $+X$ near $\eta=0$ is expected to exhibit power-law behavior in $E_{T}$ in three distinct $E_{T}$-regions with three different exponents, namely: $-5,-7$, and -9 . While the dynamical origin of such power-law behavior is SOC, the powers $\left(\varphi^{\prime}\right.$ s) in the phase-space-factors can be directly derived from first principles. That is to say, comparison between the existing data 912 and the results obtained from an analysis of the space-time properties of the colliding objects have led us to the following conclusion: The existing $p+\bar{p} \rightarrow$ jet $+X$ data9 12 do not exhibit any irregularity which could be considered as an indication for the compositness of quarks.

(III) In the search for "new state of matter", concepts and methods of conventional Equilibrium Thermodynamics play an extremely important role. In fact, it has always been taken for granted that there is thermal and chemical equilibrium in the relevant systems, and thus concepts such as temperature and chemical potential are suitable quantities for the description of the expected "new state of matter QGP" 1. I I seems that questions such as the following have never been asked: What can we do, or should we do, if systems of quarks and gluons are far beyond thermal and/or chemical equilibrium? Is there direct experimental evidence for or against the existence of thermal and/or chemical equilibrium in squeezed nucleon systems?" Based on the results presented in this paper, and the wellknown fact (See e.g. 
we think it would be very useful to measure the $E_{T}$-distributions of $(\eta \approx 0)$ jet in high-energy hadron-nucleus and nucleus-nucleus collisions, and check whether SOC-fingerprints also exist when more than two hadrons are squeezed together.

We thank F. Golcher, D. H. E. Gross, and W. Zhu for discussions and J. Terron for correspondence. 


\section{REFERENCES}

1. See e.g. U. Heinz, M. Jacob, nucl-th/0002042; and references given therein.

2. See e.g. M. Jacob, The Quark Structure of Matter (World Scientific, Singapore, 1992); and references given therein.

3. See e.g. G. C. Blazey and B. L. Flaugher, Ann. Rev. Nucl. Part. Sci. 49, 633 (1999); and references given therein.

4. P. Bak, C. Tang, and K. Wiesenfeld, Phys. Rev. Lett. 59, 381 (1987); P. Bak, C. Tang, K. Wiesenfeld, Phys. Rev. A 38, 364 (1988).

5. P. Bak, How Nature Works (Springer, New York, 1996); H. J. Jensen, Self-Organized Criticality (Cambridge University Press, Cambridge, 1998).

6. T. Meng, R. Rittel, Y. Zhang, Phys. Rev. Lett. 82, 2044 (1999).

7. C. Boros, T. Meng, R. Rittel, K. Tabelow, Y. Zhang, Phys. Rev. D. 61, 094010 (2000).

8. See e.g. H. Abramowicz, A. Caldwell, Rev. Mod. Phys. 71, 1275 (1999); A. M. CooperSarkar, R. C. E. Devenish, A. de Roeck, Int. J. Mod. Phys. A 13, 3385 (1998); and references given therein.

9. J. Appel et al., Phys. Lett. B 160, 349 (1985).

10. G. Arnsion et al., Phys. Lett. B 172, 461 (1986).

11. J. Alitti et al., Phys. Lett. B 257, 232 (1991).

12. F. Abe et al., Phys. Rev. Lett. 77, 438 (1996).

13. Similar power-law behavior has been observed, and similar conclusions can be drawn also in lepton-induced deep-inelastic scattering events. This will be discussed in detail elsewhere (in preparation, not yet published).

14. C. Albajar et al., Z. Phys. C 36, 33 (1987). 
15. E. Rutherford, Phil. Mag. XXI, 669 (1911).

16. E. J. Williams, Rev. Mod. Phys. 17, 217 (1945). 


\section{FIGURES}

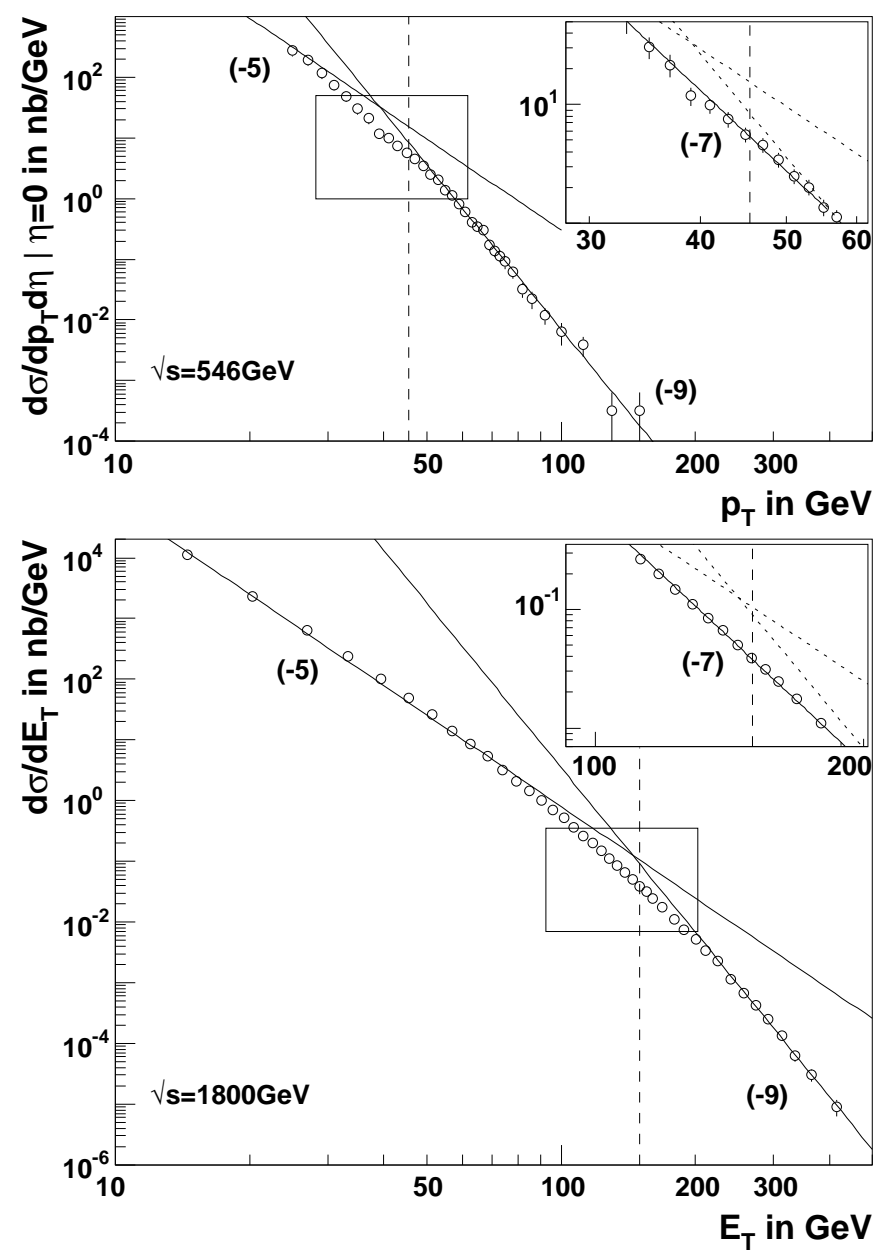

Fig. 1. Evidence for the validity of Eq.(2) for $\bar{p}+p \rightarrow$ jet $+X$ at $\eta \approx 0$ and different c.m.s energies $\sqrt{s}$. Comparisons have been made with data in Refs. [9 12]. For the sake of clearness, at $546 \mathrm{GeV}$, only data in Ref. 10] are shown in this figure. Those in Refs. [9, 11] and those at 630 $\mathrm{GeV}$ [10] exhibit the same regularities. The numbers shown in the brackets stand for the slopes of the straight lines in the log-log plots, that is, the value of $-\alpha$ in Eq.(2). The vertical dashed line indicates $E_{T}=\sqrt{s} / 12$. We note: The empirical fact that there are three $q_{v}$ 's in $p$, and about $50 \%$ of the total c.m.s. energy $\sqrt{s} / 2$ of $p(\bar{p})$ is carried by the gluons, suggest that TSV processes should begin to dominate for $E_{T}>\sqrt{s} / 12$. 


\section{TABLES}

\begin{tabular}{|c|c|c|c|c|c|c|}
\hline \multirow{2}{*}{ Process } & \multirow{2}{*}{ Step } & \multirow{2}{*}{ Participants } & \multicolumn{3}{|c|}{$\varphi=\alpha+1-2 \cdot \mu$} & \multirow{2}{*}{$E_{T}^{-\alpha}$} \\
\hline & & & $\begin{array}{l}\text { due to } \\
\Delta x \Delta y\end{array}$ & $\begin{array}{l}\text { due to } \\
\Delta t\end{array}$ & total & \\
\hline OS & & $c^{\star} \quad c^{\star}$ & 2 & & 2 & $E_{T}^{-5}$ \\
\hline \multirow{4}{*}{ TSS } & $1 \mathrm{st}$ & $c^{\star} \quad q_{s}$ & & 1 & \multirow{4}{*}{4} & \multirow{4}{*}{$E_{T}^{-7}$} \\
\hline & $2 \mathrm{nd}$ & $c^{\star}$ & 2 & 1 & & \\
\hline & 1 st & $q_{s}$ & & 1 & & \\
\hline & $2 \mathrm{nd}$ & $c^{\star}$ & 2 & 1 & & \\
\hline \multirow{4}{*}{ TSV } & $1 \mathrm{st}$ & $c^{\star}$ & 2 & 1 & \multirow{4}{*}{6} & \multirow{4}{*}{$E_{T}^{-9}$} \\
\hline & $2 \mathrm{nd}$ & $c^{\star}$ & 2 & 1 & & \\
\hline & 1 st & $q_{v} \quad q_{v}$ or $q_{s}$ & 2 & 1 & & \\
\hline & 2nd & $c^{\star}$ & 2 & 1 & & \\
\hline
\end{tabular}

Table 1. Estimation of phase-space-factors for different processes by making use of the Uncertainty Principle and the observations made in 15,16]. See text for more details. 